\title{
Polarized Fluorescence of Spin-coated Polymeric Thin Films
}

\author{
Satoko Nishiyama, Masahiro Tajima ${ }^{\dagger}$, and Yasuhiko Yoshida ${ }^{{ }^{\star \star}}$ \\ Bio-Nano Electronics Research Center and ${ }^{\dagger}$ Department of Applied Chemistry, Faculty of Engineering, \\ Toyo University, Kujirai 2100, Kawagoe-shi, Saitama, 350-8585, Japan \\ yoshida@eng.toyo.ac.jp
}

\begin{abstract}
We show an attempt of analyzing the distributions of transition moments of fluorophores in thin films. At a certain excitation wavelength, fluorophores can be excited with polarized light of different transition probabilities which are dependent on the direction of the transition moment. We compared emission bands of some pyridine-containing polymer films with varying the polarization angle of the polarizer and/or analyzer. The polarization angle dependence of these emission bands appeared to be different for different types of emission bands, and the distributions of the transition moments were discussed.
\end{abstract}

Keywords: polarized fluorescence, excitation emission matrix, transition moment, polymeric thin films, pyridine derivative

\section{Introduction}

Pyridine derivatives (Pys; 1) show various optical properties and are utilized for various purposes, such as a base, a ligand, or a "proton sponge". The complexity comes from the nature of hetero atom, nitrogen. With the lone pairelectrons of $\mathrm{N}$-atom play an important role in their photo-excited states. Piérola et al. reported the photoinduced protonation process of poly(2-vinylpyridine)(P2VPy; 2a) in the presence of some proton donors[1-5] in '80s. In those days, Handa et al. reported luminescence of protonated-pyridine derivatives (Pys- $\mathrm{H}^{+}$) [6-10]. In '90s and '00s, luminescence and metal binding properties of protonated-poly(vinylpyridines) (PVPys- $\mathrm{H}^{+}$) in acidic solutions were reported by Vyprachtický et al.[11] and luminescent behaviors of concentrated poly(4-vinylpyridine) (P4VPy; 2b) solutions in the presence of $\operatorname{Py}(1 \mathrm{a})$ were also reported by Vaganova et al.[12-15] They observed interesting gelatinization and luminescence changes in P4VPy(2b) solutions. They attributed the reason of the gelatinization of the solution to be the protonation of Py(1a) and cross-linking effect of hydrogen-bond. They also mentioned the possibility of decomposition of Py-ring[16]. We have determined luminescence and photochemical reactions of poly (vinylpyridine) (PVPys; 2) and related Pys(1)[17-
29]. In our previous reports[27-29], we showed the speculated photo-induced protonation scheme in the excited states of Pys(1) as shown in Scheme 1.

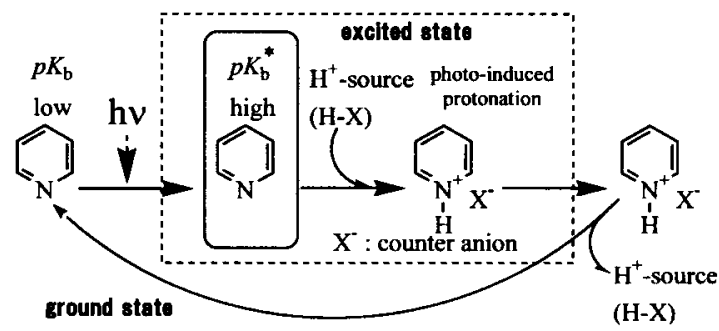

Scheme 1. Photoinduced-protonation of Py (1a)

On the other hand, we reported the incident polarization angle-dependence of PVPys(2) thin films fluorescence[25,26] using an apparatus for a solid sample holder (as shown in Scheme 2).
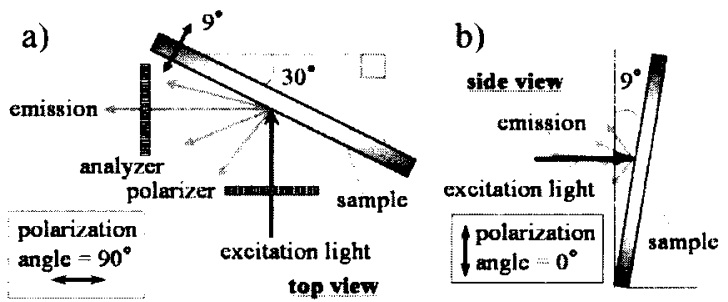

Scheme 2. The apparatus for polarization fluorescence measurement ( $a)$ : top view, and b): side view).

We calculated polarized emission intensities of thin 
films of PVPys(2) with incident polarized light correction, as a fluorescence spectrophotometer monitors the monochromated excitation light before the polarization (a photo detector for monitoring the excitation light intensity is placed ahead of the polarizer). We used three kinds of pyridine reactions, two kinds of quaternization with alkyl bromides(3) and UV-iradiation after protonation with carboxylic acid. Each reaction was quarternization of P2VPy (2a) with ethyl bromide (EtBr; 3a); (P2VPy+EtBr (4a)), quaternization of P4VPy (2b) with cyclopropyl bromide (cyc- $\mathrm{PrBr}$ (3a)) $(\mathrm{P} 4 \mathrm{VPy}+c y c-\operatorname{PrBr}(\mathbf{4 b}))$, or the UV-irradiation after the protonation of $\mathrm{P} 4 \mathrm{VPy}(\mathbf{2 b})$ with formic acid (FA; 5); (P4VPy+FA(4c)+UV), respectively. We found that the incident-polarization angle dependence of each reaction is a little bit different from each other. But unfortunately, we couldn't make corrections for emission side-optical path (the anisotropy of the analyzer, the monochromator, and the photomultiplier) in our previous report. The almost all the calculated incident polarization-angle dependence of modeified-PVPys(4) films showed similar features[26], decrease with increasing incident polarization angle. In this study, we show an attempt of further correction for the determination of the polarized emission intensities.

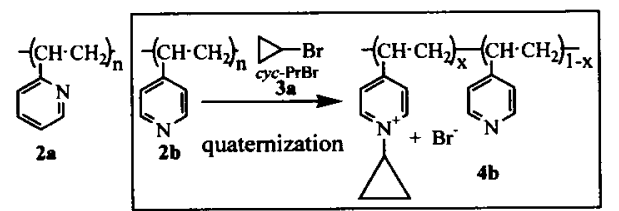

Scheme 1. Structures of P2VPys(2a), P4VPy(2b) and quaternized reaction of $\mathrm{P} 4 \mathrm{VPy}(\mathbf{4 b})$.

\section{Experimental}

\subsection{Materials and sample preparation}

P2VPy(2a) and P4VPy(2b) were the products of Sigma-Aldrich Corp. and we purchased them and used as received. Both alkyl bromides(3), EtBr (3a) $(98.0 \%$, Kanto Chemicals Co., Inc.) and cyc$\operatorname{PrBr}(3 \mathbf{b})$ (Tokyo Kasei Kogyo Co.,Ltd.) were also purchased and used without further purification. Methanol (Dojindo Molecular Technologies, Inc.) was of spectral grade, and FA (Wako Pure Chemicals Co.) was of reagent grade.

\subsection{Quaternization process}

Thin films of PVPys(2), spin-coated on quartz substrates were placed inside of a pair of closed glass dishes with a small bottle of an alkyl bromide (3). Quaternization was achieved with vaporized alkyl bromide (3). Progress of quaternization was confirmed by changes in XPS N1s peaks $[19,20,29]$.
Details were described elsewhere[19-21,23,25,26].

2.3. UV-irradiation process

P4VPy+FA(4b) or P4VPy(2b) was irradiated using a small $\mathrm{Hg}$ lamp at $254 \mathrm{~nm}$ mode[22-29].

2.4. Absorption and luminescence measurements

Absorption spectra of P4VPy(2b) films were measured with a Hitachi U-3500 spectrophotometer. All EEMs (excitation emission matrices) and emission spectra [19-29] were recorded with a Hitachi F-4500 Fluorescence spectrophotometer with polarization units. We use the expression $\operatorname{Em}\left(\lambda_{\text {ex }}, \lambda_{\text {em }}\right)$ for the maximum or typical wavelengths of each emission band[19-29]. Because of the strong scattering on solid surfaces, correct values of the emission bands were difficult to discriminate. Usually $\lambda_{\mathrm{ex}}$ is varied in the range of $220 \sim 600 \mathrm{~nm}$ and $\lambda_{\mathrm{em}}$ is $220 \sim 800 \mathrm{~nm}$. To obtain correct emission spectra, we measured light intensities in the optical path of F-4500 by an Ophir Nova energy meter (we abbreviate it to "EM") equip with a photo detector(PD-300-UV). Details are described in chapter 3.

2.5. Luminescence classifications

We classified the observed emission bands in EEMs into four types [25, 26](see Fig. 1 and Table 1).

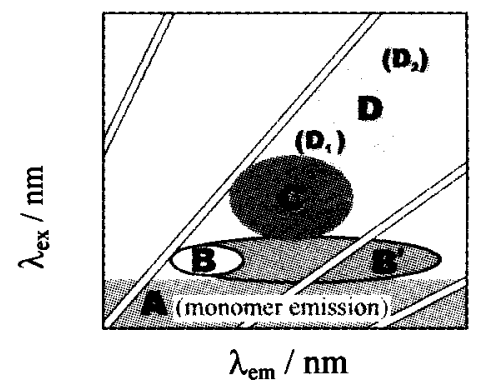

Figure 1. Schematic representation of the speculated classification of emission bands in EEMs for PVPy(2) (vertical axis : excitation wavelength $\left(\lambda_{\mathrm{ex}}\right)$, horizontal axis : emission wavelength $\left.\left(\lambda_{\mathrm{em}}\right)\right)$

Table 1. Emission bands of PVPys(2)

\begin{tabular}{|c|c|}
\hline $\begin{array}{c}\text { Emission } \\
\text { bands }\end{array}$ & $\begin{array}{c}\text { Speculated classification of each } \\
\text { emission band }\end{array}$ \\
\hline $\mathbf{A}$ & $\begin{array}{c}\text { monomer emission }\left(\pi-\pi^{*} \text { excitation }\right) \text { of } \\
\text { pyridine-rings }\end{array}$ \\
\hline $\mathbf{B}$ & $\begin{array}{c}\text { monomer emission ( } \mathrm{n}-\pi^{*} \text { excitation) of } \\
\text { pyridine rings or } \\
\text { protonated / quaternized ionic species }\end{array}$ \\
\hline $\mathbf{B}^{\prime \ddagger}$ & $\begin{array}{l}\text { CT-like-emission from higher excited } \\
\text { states }\end{array}$ \\
\hline $\mathbf{C}$ & emission from hydrogen-bonded species \\
\hline D & $\begin{array}{c}\text { emission from CT-complexes formed in } \\
\text { the ground state }\end{array}$ \\
\hline
\end{tabular}

${ }^{\ddagger}$ Bands $B$ and -B' are excited at same $\lambda_{\text {ex. }}$.

In EEMs, almost all emission bands appear between 
two Reileigh scattering lines (they appear at $\lambda_{\text {ex }}$ and $2 \times \lambda_{\text {ex }}$, and other harmonic $\lambda$ ). Type $A$ band is nearly impossible to analyze because of the high absorbance and scattering of sample films.

\section{Corrections for anisotropy of the apparatus}

To compare spectra at any $\theta, \phi$ and $\lambda$, the following correcting procedures are necessary [30].

\subsection{Corrections for polarization (excitation side)}

Correction procedure of excitation process was discussed in our previous reports [26,27].

A $150 \mathrm{~W}$ Xe-lamp is used for the excitation light. Its intensity changes with transmitting through the polarizer and other optical devices. Usually, the output signal $\left(E_{1}\right)$ is provided after division of the emission light intensity $\left(I_{1}\right)$ by the monochromated source light intensity monitored with a built-in photo detector $\left(I_{\mathrm{PD}}\right)$. At a certain wavelength $(\lambda$ $\mathrm{nm})$, the excitation light $\left(I_{\mathrm{ex} 0}\right)$ and the polarized light intensity with a certain polarization angle $(\theta)$ $\left(I_{\mathrm{ex}}(\theta, \lambda)\right)$ can be expressed with a function $\alpha(\theta, \lambda)$,

$I_{\mathrm{ex}}(\theta, \lambda)=\alpha(\theta, \lambda) \times I_{\mathrm{ex} 0}(\theta, \lambda)$

The correction matrix $\alpha(\theta, \lambda)$ used in this study is shown in Fig2 (same to that used in our previous report[26]), and Fig.3a) and 3b) (appears in 3.3.) depicts the relationships between the light intensity and correction matrices for optical devices.

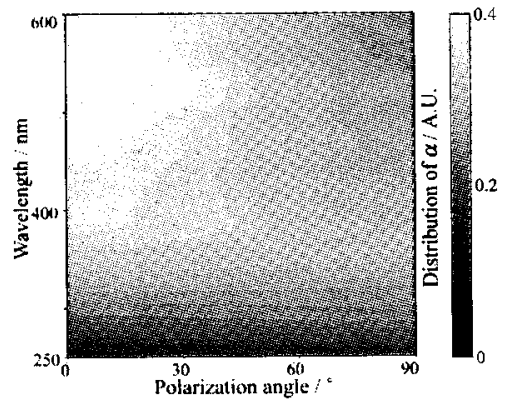

Figure 2. Distribution of $\alpha(\theta, \lambda)$

(vertical axis : excitation wavelength $\left(\lambda_{\text {ex. }} / \mathrm{nm}\right)$, horizontal axis : incident polarization angle $/{ }^{\circ}$ )

\subsection{Rotation angles $(\theta, \phi)$ of polarizers}

In usual experiment, as the medium is treated to be an isotropic one for fluoresce molecules, we don't need to distinguish the rotating direction $( \pm)$ of $\theta$ or $\phi$ to be clockwise or counterclockwise. In this study, as the incident angle of the excitation light on the film is tilted, we have to distinguish \pm of the rotating direction. So we define + direction as the counterclockwise in the direction viewing each monochromator $\left(\mathrm{M}_{\mathrm{ex}}\right.$ or $\left.\mathrm{M}_{\mathrm{em}}\right)$ from the sample holder (as shown in Fig.3c) in 3.3.). The rotation angle of the excitation light described in our previous study was opposite to $\theta$ in Fig.3c) $(\theta=-\chi$ in ref. 26), in the range of $0^{\circ} \leq \chi \leq 90^{\circ}$. (Details are described later, in 3.4.) So the equation of the $p$ - and $s$-components (in-plane and perpendicular components) of the incident light as follows,

$$
\begin{aligned}
I_{\mathrm{x}}= & \left((q \sin \beta-p)^{2}+(q \cos \beta)^{2}\right) \times 0.5 I_{\mathrm{p}} \\
& +\left(\cos \theta \sin 9^{\circ}+\sin \theta \cos 9^{\circ} / 2\right) I_{\mathrm{s}}
\end{aligned}
$$

( $p, q, \beta$ : described in ref. 26).

Therefore, from eq. (2), when $\tan \theta=-2 \times \tan 9^{\circ}$, $s$-component of the light is equal to 0 . Then, $\theta$ can be calculated to $\sim 17.6^{\circ}$.

It means that $I_{\mathrm{x}}=\left((q \sin \beta-p)^{2}+(q \cos \beta)^{2}\right) \times 0.5 I_{\mathrm{p}}$ when $\theta \sim 17.6^{\circ}$.

\subsection{Corrections for polarization (emission side)}

For the compensations for instrumental functions, several functions are defined and the relationships among them are depicted in Fig.3.
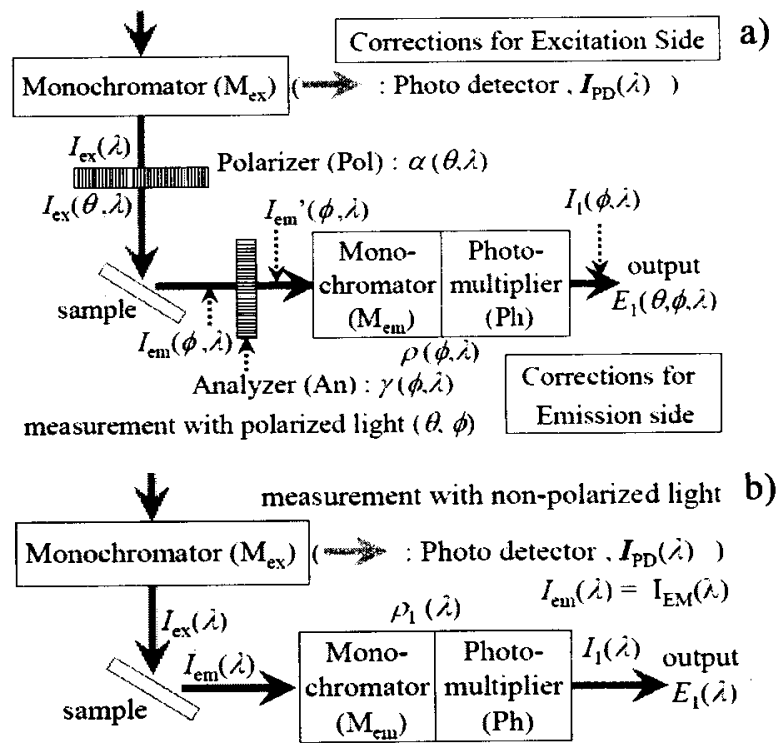

b)
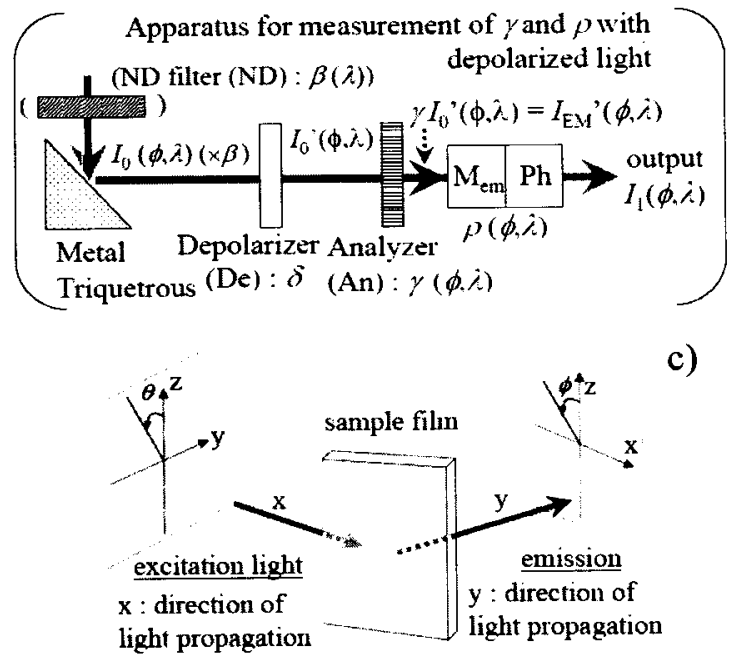

c)

Figure 3. Schematic representations of the setup for the determination of the emission light intensity.

(Each abbreviation is described in the text.)

The instrumental functions for Pol, ND filter (ND), depolarizer (De), analyzer (An) (the polarizer in the emission side, with polarization angle $\phi$ with respect to the vertical axis), $\mathrm{M}_{\mathrm{em}}$ and photo- 
multiplier (Ph) are defined as $\alpha(\theta, \lambda), \beta(\lambda), \delta(\lambda)$, $\chi \phi, \lambda)$ and $\rho(\phi, \lambda)$, respectively. $\gamma$ and $\rho$ are defined as instrumental functions, which consist of two parts, $\gamma_{1}, \rho_{1}$ ( $\phi$-independent components) and $\gamma_{2}$, $\rho_{2}$ ( $\phi$-dependent components), respectively. When the light is not polarized, $\gamma=\gamma_{1}$ and $\rho=\rho_{1}$. Besides, they are equal to transmittance.

\subsubsection{Calculation and determination of $\gamma$}

With non-polarized light, we obtain the signal $E_{1}$ using a F-4500 fluorescence spectrophotometer. It is represented as follows,

$E_{1}=I_{1} / I_{\mathrm{PD}}$

$I_{1}$ can be represented as

$I_{1}=\rho \times I_{\mathrm{em}}=\rho_{1} \times I_{\mathrm{em}}$

Hence,

$I_{\mathrm{em}} / I_{\mathrm{PD}}=I_{1} / \rho_{1} / I_{\mathrm{PD}}=E_{1} / \rho_{1}$

where $I_{\mathrm{em}}$ is the emission intensity which is observed in the direction of the detector.

If we measure the light intensity by EM in the front of $M_{e m}$, the observed light intensity $I_{\mathrm{EM}}$ can be described by the product of the multiplication of $I_{\mathrm{em}}$, which emerged from irradiated sample, by the correction matrices.

With polarized light, we measure the signal intensity $E^{\prime}$ as follows,

$E^{\prime}=I_{1}^{\prime} / I_{\mathrm{PD}}$

here $I_{1}{ }^{\prime}$ is the corresponding output intensity of $\mathrm{Ph}$.

If we define $I_{\mathrm{em}}$ ' as the emission intensity with polarized light,

$I_{1}{ }^{\prime}=\gamma \rho \times I_{\mathrm{em}}{ }^{\prime}$

$I_{\mathrm{em}}{ }^{\prime} /\left(\alpha I_{\mathrm{PD}}\right)=I_{1}{ }^{\prime} /(\gamma \rho) /\left(\alpha I_{\mathrm{PD}}\right)=E^{\prime} /(\alpha \gamma \rho)$

To determine the correct emission intensity per excitation light, we have to divide $E^{\prime}$ by $\alpha \gamma \rho$. Both correction matrices, $\chi(\phi, \lambda)$ and $\rho(\phi, \lambda)$ were determined prior to the fluorescence measurement.

To obtain $\gamma_{1}$ and $\gamma_{2}$ of $\chi(\phi, \lambda)$, at a certain wavelength $(\lambda \mathrm{nm})$, we determined the Xe-lamp light intensity $I_{0}(\phi)$ (reflected by a polished surface of a metal triquetrous after monochromated by $\mathbf{M}_{\mathrm{ex}}$ ) and $I_{0}{ }^{\prime}(\phi)$ is the reflected light intensity after permeation through De. $\beta(\lambda)$ and $\delta(\lambda)$ are the compensation function for ND(it was used only for the preparative determination of $I_{1}(\phi)$ using F-4500) and $\mathrm{De}$ (it is also used only for the preparative determination), which were drawn in a parenthesis in lower part of Fig.3b). $\quad(\lambda)$ is equal to transmission spectrum of ND. EM detected the light intensity $\left(I_{\mathrm{EM}}\right)$ when the reflected monochromated light $\left(I_{0}\right)$ was irradiated on it.

$I_{\mathrm{EM}}$ correspond to $I_{0}$ with non-polarized light ( $I_{0}$ determined without An).

The light intensity between De and An is equal to $\delta$ $\times I_{0}$. Therefore, $I_{1}$ detected by $\mathrm{Ph}$ is
$I_{1}=\delta \times \rho_{1} \times \beta \times I_{0}$

$I_{1}^{\prime}=\delta \times \gamma \times \rho \times \beta \times I_{0}$

Simultaneously, in the rear of An, the light intensity $I_{0}{ }^{\prime}(\phi)$ is changed to $I_{\mathrm{EM}}(\phi, \lambda)$, and $I_{\mathrm{EM}}$ ' is equal to $\chi(\phi, \lambda) \times I_{0}{ }^{\prime}(\phi, \lambda)$. With polarized light, $I_{\mathrm{EM}}(\lambda)$ and $I_{\mathrm{EM}}(\phi, \lambda)$ detected by EM are described as follows,

$$
\begin{aligned}
& I_{\mathrm{EM}}=\delta I_{0} \\
& I_{\mathrm{EM}}=\delta \gamma I_{0}, \\
& I_{\mathrm{EM}} / I_{\mathrm{EM}}=\delta \gamma I_{0} / \delta I_{0}=\gamma
\end{aligned}
$$

It was difficult to obtain accurate value of $\left(I_{\mathrm{EM}}{ }^{\prime} / I_{\mathrm{EM}}\right)$ in a good $\mathrm{S} / \mathrm{N}$ ratio, because of the strong sharp line of the Xe-lamp. So they were measured separately in the actual measurement in the following procedures. The obtained $\chi(\theta, \lambda)$ is shown in Fig.4.

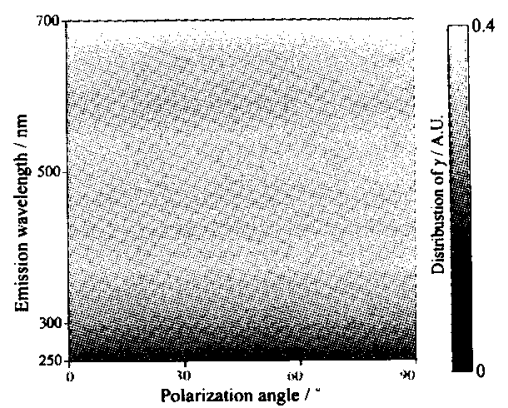

Figure 4. Distribution of $\chi(\phi, \lambda)$ (vertical axis : excitation wavelength $\left(\lambda_{\text {ex. }} / \mathrm{nm}\right)$, horizontal axis : An polarization angle $\phi /^{\circ}$ )

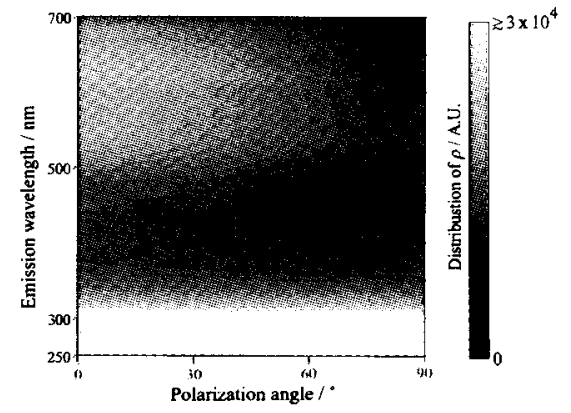

Figure 5. Distribution of $\rho(\phi, \lambda)$

(vertical axis : excitation wavelength $\left(\lambda_{\text {ex }} / \mathrm{nm}\right)$, horizontal axis : An polarization angle $\phi /{ }^{\circ}$ )

Absorption spectrum $\left(\phi=45^{\circ}\right)$ of An was used as $\gamma_{1}$. $\gamma_{2}$ was measured as the intensity change of $I_{\mathrm{EM}}$ according to $\phi$. We defined $\gamma_{2}$ as the ratio between $I_{\mathrm{EM}}(\phi)$ and $I_{\mathrm{EM}}{ }^{\prime}\left(\phi=45^{\circ}\right)$.

$\gamma_{2}=I_{\mathrm{EM}}(\phi) / I_{\mathrm{EM}}{ }^{\prime}\left(\phi=45^{\circ}\right)$

In general, as $\phi$-dependence in $\gamma$ is low, the influence of $\gamma$ should be smaller than $\alpha$ and $\rho$.

3.3.2. Calculation and determination of $\rho$

Not only $\gamma$, a difficulty was also found in the measurement of $\rho(\phi, \lambda)$. Because of each monochromatism of the incident light on EM or $\mathrm{Ph}$ is different, we used smoothing procedures for the values of $I_{\mathrm{EM}}$ ' and $I_{1}$. From eqs. (9) and (11), 
$\frac{I_{\mathrm{EM}}{ }^{\prime}(\phi)}{\delta(\lambda) \times \chi \phi, \lambda)}=\frac{I_{1}^{\prime}(\phi)}{\chi(\phi, \lambda) \times \delta(\lambda) \times \beta(\lambda) \times \rho(\phi, \lambda)}$

$\therefore I_{1}{ }^{\prime}(\phi) / I_{\mathrm{EM}}{ }^{\prime}(\phi)=\beta(\lambda) \rho(\phi, \lambda)$

$\rho(\phi, \lambda)$ was calculated as $\left(I_{1}{ }^{\prime} / I_{\mathrm{EM}} / \beta\right) . \rho(\phi, \lambda)$ is expressed in Fig.5. From the values of $\gamma$ and $\rho, \lambda$ $\leq 300 \mathrm{~nm}$ would be difficult to calculate precisely (See $\lambda \leq 300 \mathrm{~nm}$ regions of Fig. 4 and Fig.5.

3.4. Calculations of emission polarization angle $(\phi)$ dependence of the emission intensity

We discussed the excitation polarization angle $(\theta)$ dependence in our previous report[26]. In this study, we consider the emission polarization angle in a similar manner. The Analyzer (An) has a rotation angle $\phi$ (see Fig.3c)) and it is equal to the polarization angle. As the sample substrate has an $\sim 81^{\circ}$ elevating angle, we use new axis of coordinates rotated $\phi_{0} \sim-10.3^{\circ}$ from the vertical line. We can define new rotation angle $\phi^{\prime}=\phi+\phi_{0}$. At $\phi^{\prime}=0^{\circ}$, the intersection line between the sample film and the surface of An is parallel to the polarization direction. It means that all of the transmitted light is in-plane ( $p$-component), and the $p$-component should decrease with increasing $\phi$. Therefore, we can obtain information about the $p$ and $s$-components $\left(i_{\mathrm{p}}, i_{\mathrm{s}}\right)$ of the fluorophores' transition moments in their excited states with $\phi$ dependence of fluorescence intensity.

$i_{\phi}=\cos \phi \times i_{(\phi=0)}+\sin \phi \times i_{(\phi=90)}$

$=\cos \phi \times i_{\mathrm{p} 1}+\sin \phi \times\left\{\left(3^{0.5} / 2\right) \times i_{\mathrm{p} 2}+(1 / 2) \times i_{\mathrm{s}}\right\}$

$P$-component $\left(i_{\mathrm{p}}\right)$ of $i_{\phi}$ is calculated as follows, $\left|i_{\mathrm{p}}\right|=\left|\left(i_{\mathrm{p} 1}\right)^{2}+\left(i_{\mathrm{p} 2}\right)^{2}\right|$

$$
=\left(\cos ^{2} \phi^{\prime}+(1 / 2)^{2} \times \sin ^{2} \phi^{\prime}\right)^{0.5} \text {. }
$$

and s-component $\left(i_{\mathrm{s}}\right)$ of $i_{\phi}$ can be calculated as

$\left|i_{\mathrm{s}}\right|=\left(\left(3^{0.5} / 2\right)^{2} \times \sin ^{2} \phi^{\prime}\right)^{0.5}$.

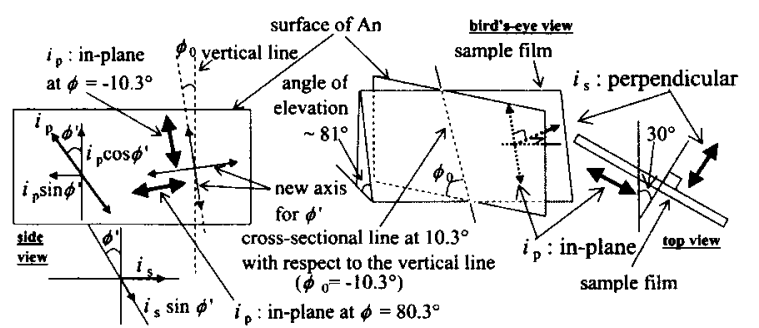

Figure 6. Schematic representations of the relationships among $\phi, \phi_{0}, \phi$ and surfaces of An and the sample film.

Eqs. (18) and (19) mean that $p$ - or $s$-polarized emission light is converted into $\phi$-polarized light with the ratio of the above equation, respectively. With these eqs., $p$ - and $s$-components of emission light were calculated, as shown in Fig.7a).

As described in 3.2., $p$ - and $s$-components of the excitation light were reported previously[26]. We have to correct those components' change with $\theta(r$ and $s$ in Fig.6 in ref.26) as $p / \theta$ and $s / \theta$ in Fig.7a).
In Fig.7b), products of each (excitation and emission side) $p$ - or $s$-components are also shown. As the sample films weren't made to align to a certain direction, sample films probably contain multiple systems. As an example, relative intensity changes of the 1:1 mixture of two systems are drawn in Fig.7c). As shown in Fig.7c), systems which contain $p \times p$ keeps high intensity especially in the range of $\theta$ or $\phi$ is small. Contrary to it, systems which don't contain $p \times p$ have high intensities only in the range of $\theta$ or $\phi$ is large.
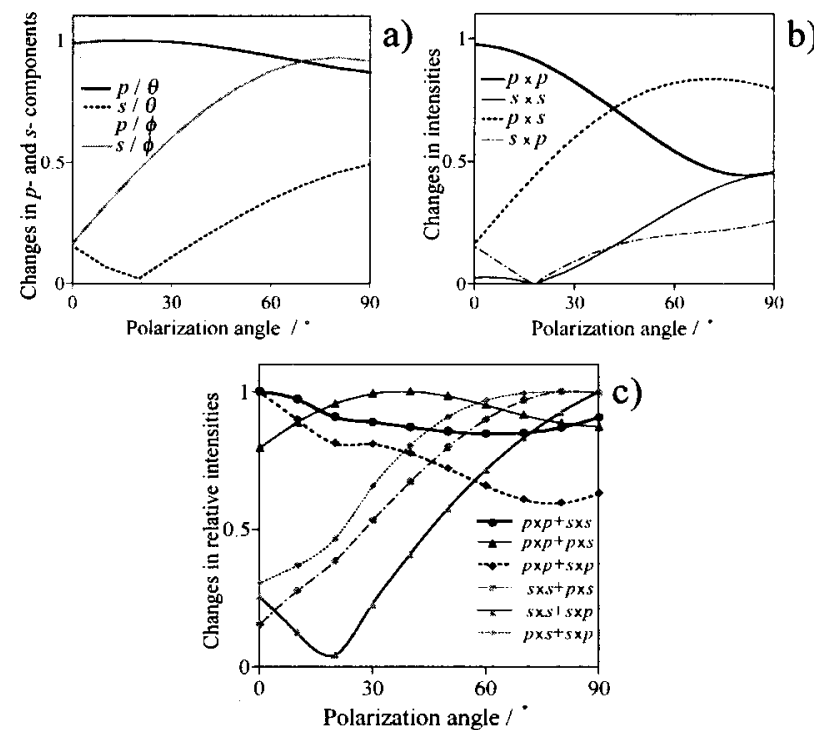

Figure 7. Changes in $p$ - and $s$-components with $\theta$ or $\phi$.

(a): each $p$ - and $s$-components, b): changes in the products of two (excitation / emission side) components, $\theta=\phi$.) c): relative intensities of $1: 1$ mixture.

\section{Results and Discussion}

With the corrections described in the previous section, the corrected polarization dependence of fluorescence is displayed and discussed in the following sentences. The progress of the quaternization of PVPys(2) were confirmed with XPS in our previous reports $[19,20]$. P4VPy(2b) films were UV-irradiated with no addition of external proton-donors, and their luminescence were measured with or without polarizers.

4.1. Emission intensity correction of quaternizedPVPys(4) with polarization angles (at $\theta=\phi$ )

The changes in the fluorescence intensities of $\mathrm{P} 4 \mathrm{VPy}+c y c-\operatorname{PrBr}(\mathbf{4 b})$ are shown in Fig.7a). Two broad emission bands $\left(F_{1}(\bullet)\right.$ and $F_{2}(\circ)$, shown in Fig.7a), respectively) are seen for $\mathrm{P} 4 \mathrm{VPy}+c y c-\mathrm{PrBr}$ (4b). $\quad F_{1}$ is seen at longer $\lambda_{e x}$ and $F_{2}$ is at shorter $\lambda_{\text {ex }}$. The emission wavelengths of both emission bands are close. Especially, band $F_{1}$ appeared to be smaller for larger $\theta$. Fluorescence change in $\mathrm{P} 2 \mathrm{VPy}+\mathrm{EtBr}(\mathbf{4 a})$ was also calculated with both 
corrections. The results are depicted in Fig.7b). $\mathrm{P} 2 \mathrm{VPy}+\mathrm{EtBr}(\mathbf{4 a})$ also has two emission bands $\left(\mathrm{F}_{3}\right.$ $(\bullet)$ and $F_{4}(O)$, respectively) at different $\lambda_{\mathrm{em}}$.
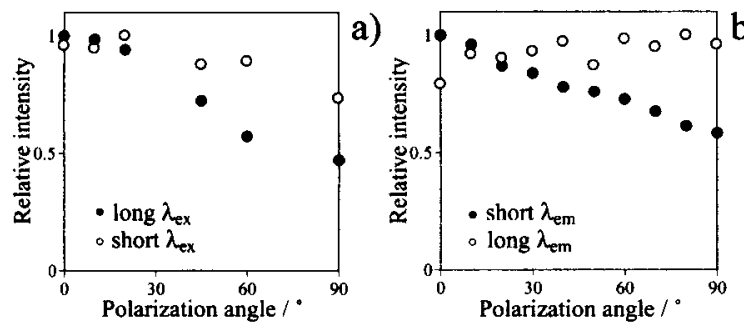

Figure 8. Changes in quaternized-PVPys $(\mathbf{4 a}, \mathbf{4 b})$ fluorescence with polarization angle $\theta=\phi$.

(vertical axis : Relative intensity of fluorescence, horizontal axis : the polarization angle $/{ }^{\circ}$ )

(a) P4VPy+cyc-PrBr(4b); $\bullet$ : long $\lambda_{\text {ex }}, 0$ : short $\lambda_{\text {ex }}$

b) P2VPy+EtBr(4a); $\bullet$ : short $\lambda_{\text {em }}, 0$ : long $\lambda_{\text {em. }}$ )

As excited wavelength is same for both two bands, the first-excited state should be same and these two bands should compete together while their photoexcitation. As only $F_{3}$ is similar to the emission band of original P2VPy(2a), other bands should be originated from some products of quaternizedPVPys $(\mathbf{4 a}, \mathbf{4 b})$. And $\mathrm{F}_{3}$ also shows linear decrease with increasing $\theta$, though $\mathrm{F}_{4}$ appears constant at $\theta$.

These similarities between $F_{1}$ and $F_{3}$, or $F_{2}$ and $F_{4}$ agree with the similarity of the expected structures of complexes formed in quaternized-PVPys $(\mathbf{4 a}, \mathbf{4 b})$.

4.2. Corrected emission intensity of protonated$\mathrm{P} 4 \mathrm{VPy}(\mathbf{4 c})+\mathrm{UV}$ with polarization angles (at $\theta=\phi$ )

A different case of modified-PVPys(4), P4VPy $+\mathrm{FA}(\mathbf{4 c})+\mathrm{UV}$ (not quaternization but protonation with some proton sources) is shown in Fig.8a). Three emission bands (named as $F_{5}, F_{6}$, and $F_{7}$ ) are seen in EEMs of P4VPy+FA(4c)+UV (shown in Fig.9a). $\quad F_{5}(\bullet)$ and $F_{6}(0)$ slightly decrease with increasing $\theta$ and they have some resemblance to $F_{2}$ and $F_{4}$. Only $F_{7}(*)$ appeared to increase with increasing $\theta$. Only this band is fitted for the cases of no $p \times p$ concerning system (shown in Fig.7b) or 7c)). $\mathrm{F}_{7}$ has a possibility to have relationships with two different types of components, $p$ and $s$. As $\theta$ dependence of $\mathrm{F}_{7}$ was classified to $s \times p+p \times s$ type, the transition moments of the ground state and the emission state would be orthogonal. However, as the emission intensities were weak, comparison of the emission intensities should not be correct. More precise measurement would be desirable.

Judging from these three products of PVPys(2), the tendencies of these relative emission intensity with $\theta(=\phi)$ would have some possibilities to be an indicator of the nature of the emission band. The outlines of ground- and excited-state transition moments would be deduced. Transition energy levels of three modified-PVPys(4) were calculated from $\operatorname{Em}\left(\lambda_{\text {ex }}, \lambda_{\text {em }}\right)$, and they are lined in Fig.9b).
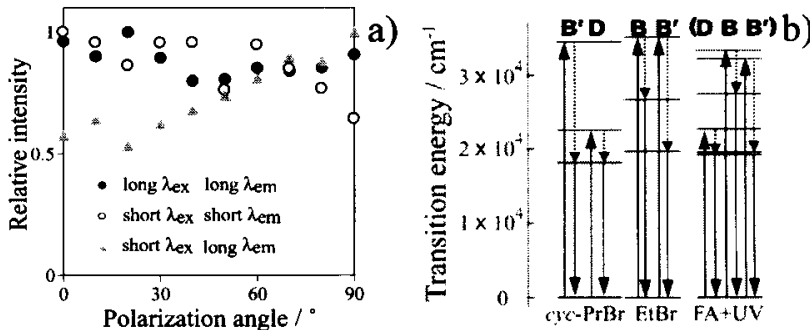

Figure 9. a) Changes in UV-irrad-P4VPy+FA(4c) fluorescence with $\theta(=\phi)$, and b) comparisons of energy levels of each emission bands.

(a): vertical axis : Relative intensity of fluorescence, horizontal axis : the polarization angle $/^{\circ}, \bullet$ : longer $\lambda_{\mathrm{ex}}$,

$\lambda_{\mathrm{em}}, \circ$ : shorter $\lambda_{\mathrm{ex}}$ and $\lambda_{\mathrm{em}}$, and : shorter $\lambda_{\mathrm{ex}}$ and

longer $\lambda_{\text {em }}$, numbered $F_{5}, F_{6}$, and $F_{7}$, respectively. )

4.3. Features of modified-PVPys(4) emission bands

Each quaternized-PVPys $(\mathbf{4 a}, \mathbf{4 b})$ film has a different tendency. The characteristics of them are listed in Table 2. For bands $F_{1}, F_{3}, F_{5}$ and $F_{6}$, the energy difference between $\lambda_{\mathrm{ex}}$ and $\lambda_{\mathrm{em}}$ are small and the first- and the final-excited electronic state which cause luminescence could be same for these cases. Among above four bands, $F_{1}$ and $F_{3}$ show significant decrease. On the other hand, $F_{5}$ and $F_{6}$ show no significant decrease.

Table 2. Emission bands of modified-PVPys(4)

\begin{tabular}{|c|c|}
\hline $\begin{array}{l}\text { Emission } \\
\text { bands }\end{array}$ & $\begin{array}{l}\text { Emission band - change with } \\
\text { increasing } \theta \text {-speculated type }\end{array}$ \\
\hline P4VPy+ & $\mathrm{F}_{1}:$ long $\lambda_{\mathrm{ex}}-\operatorname{dec}^{\dagger}-s \times s+p \times p$ ? \\
\hline$y c-\operatorname{PrBr}(\mathbf{4 b})$ & $\mathrm{F}_{2}:$ short $\lambda_{\mathrm{ex}}-\operatorname{dec}^{\dagger} ?-p \times p+s \times s ?$ \\
\hline $2 \mathrm{VPy}+\mathrm{EtBr}$ & $\mathrm{F}_{3}:$ short $\lambda_{\mathrm{em}}-\operatorname{dec}^{\dagger}-p \times p+s \times p$ ? \\
\hline (4a) & $\mathrm{F}_{4}:$ long $\lambda_{\mathrm{em}}-$ const $?-$ random? \\
\hline & $\mathrm{F}_{5}$ : long $\lambda_{\mathrm{ex}, \mathrm{em}}-\mathrm{dec} ?-p \times p+s \times s+\alpha$ ? \\
\hline $4 \mathrm{VPy}+\mathrm{FA}(4 \mathrm{c})$ & $\mathrm{F}_{6}:$ short $\lambda_{\mathrm{ex}, \mathrm{em}}-\mathrm{dec}^{\dagger}-p \times p+s \times p+\alpha$ ? \\
\hline & $\mathrm{F}_{7}:$ short $\lambda_{\mathrm{ex}}$ \\
\hline & long $\lambda_{\mathrm{em}}-$ inc $^{\ddagger}-s \times p+p \times s+\alpha$ ? \\
\hline
\end{tabular}

${ }^{\dagger} \mathrm{dec}$ : decrease, ${ }^{\ddagger}$ inc : increase, $\alpha$ : other factor ?

It has a possibility to have different origins from others, i.e., the mixture of protonated- or some products of $\mathrm{P} 4 \mathrm{VPy}(\mathbf{2 b})$ and residual $\mathrm{P} 4 \mathrm{VPy}(\mathbf{2 b})$ moiety. $F_{2}$ and $F_{4}$, which have large transition energy difference, appeared to be different from $\mathrm{F}_{7}$. Small $\theta$-dependence of them would imply the intermolecular energy transfer / migration. Only $\mathrm{F}_{7}$ showed increase, accompanied to $\theta$. It could have relationships between Py-ring and FA(5), e.g. complex formation or other processes are guessed. 4.4. Polarization angle dependence of corrected UV-irrad-P4VPy(2b) emission spectra with polarization angles (at $\theta=\phi, \theta$ or $\phi$ ) 


\subsubsection{UV-irrad-P4VPy(2b) absorption spectrum}

We have observed UV-irradiation effects on PVPys (2) in solid state and in the conjugates with some carboxylic acids in our previous reports [21-29]. To examine another type of PVPys(2) reactions, polarized fluorescence of UV-irrad$\operatorname{P} 4 \operatorname{VPy}(\mathbf{2 b})$ with no addition of external proton sources are measured. As shown in Fig.10, UVirrad-P4VPy(2b) showed new absorption peaks, a shoulder at $\sim 300 \mathrm{~nm}$ and a peak at $\sim 400 \mathrm{~nm}$.

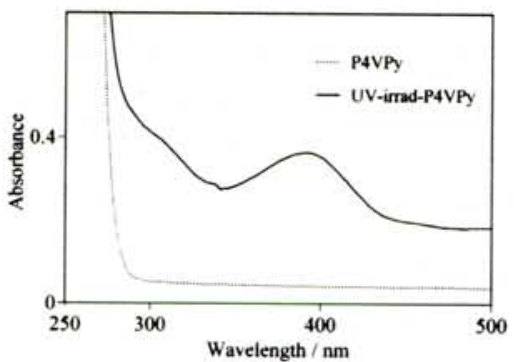

Figure 10. Absorption changes of P4VPy(2b) with UV-irradiation.
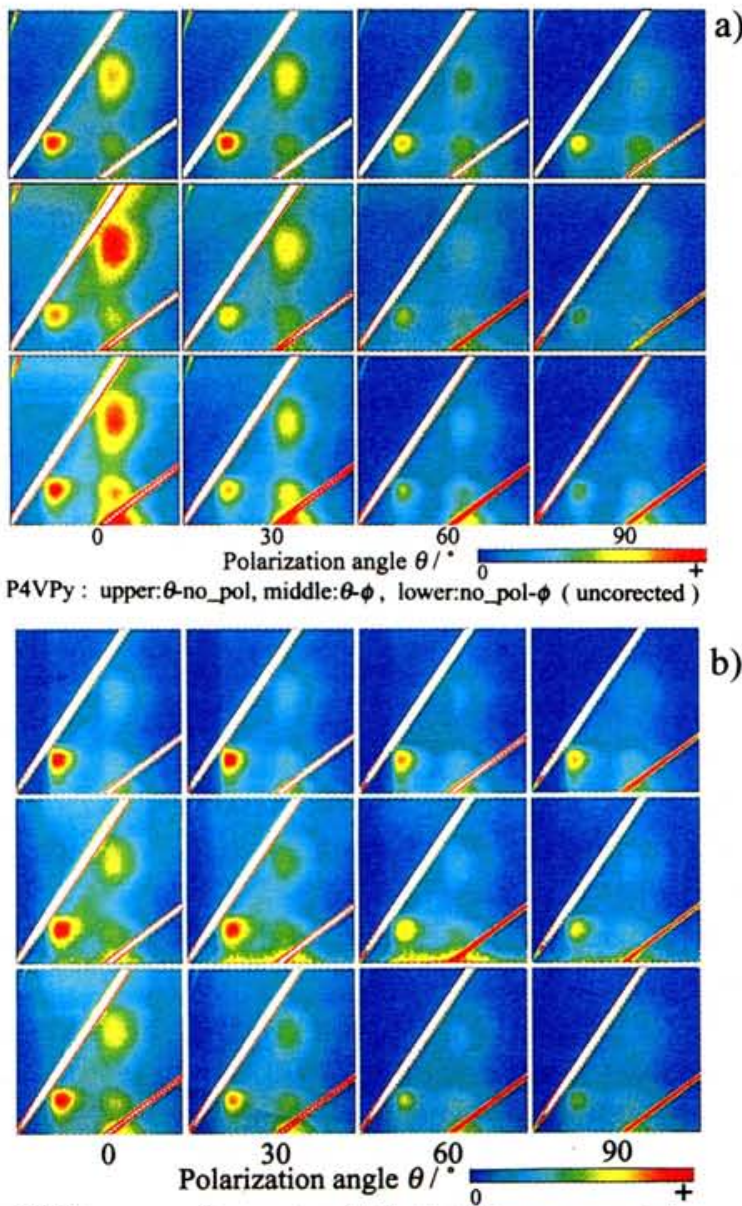

P4VPy : upper: $\theta$-no_pol, middle: $\theta-\phi$, lower:no_pol- $\phi$

Figure 11. Changes in fluorescence intensities of UV-irrad-P4VPys(2b) thin films with $\theta$ and/or $\phi$.

( $\lambda_{\text {ex }}: 250 \sim 550 \mathrm{~nm}, \lambda_{\text {em }}: 250 \sim 700 \mathrm{~nm}$, upper: $\theta$, middle: $\theta$ and $\phi$, lower: $\phi$, without (a)) or with (b)) correction)

Though there is no information of the nature of the photoproducts of PVPys(2) yet, some differences have been observed[21-29] and one possibility of the newly appeared emission band is photo-induced protonation (described in section 1) and another possibility is the decomposition of pyridine [16].

4.4.2. Incident polarization angle $(\theta)$ dependence of emission bands of UV-irrad-P4VPy(2b)

EEMs were determined and shown in Fig.11 with or without corrections described in section 3 . Three emission bands are seen and named as $\mathrm{F}_{8}, \mathrm{~F}_{9}$, and $F_{10}\left(F_{8}\right.$ : long $\lambda_{\text {ex }}$ em $; F_{9}$ : short $\lambda_{\text {ex,em }} ; F_{10}$ : short $\lambda_{\text {ex }}$, long $\lambda_{\text {em }}$; typically, $\operatorname{Em}(440,510), \operatorname{Em}(310,370)$, and $\operatorname{Em}(300,510))$, respectively. All of them were found to decrease with increasing $\theta$ and/or $\phi$. From the differences between Fig.11a) and 11b), the effect of corrections are apparent. Changes of relative intensities are depicted in Fig.12. Without corrections, changes of emission intensities (Fig. 12a)) are different from Figs.12b) 12d).

Similar to the characteristics of most of other modified-PVPys(4), emission intensities decrease with increasing $\theta$. Though $\mathrm{F}_{8}$ and $\mathrm{F}_{10}$ are found to have closer relationships with $\phi$ than $\theta, \mathrm{F}_{9}$ shows the opposite feature. In this case, $\mathrm{F}_{8}$ and $\mathrm{F}_{10}$ have same $\lambda_{\text {em. }}$, contrary to those of modified-PVPys(4). These tendencies could be related to the alignment and the difference of their nature. It is very interesting feature which have possibility of more detailed analysis.
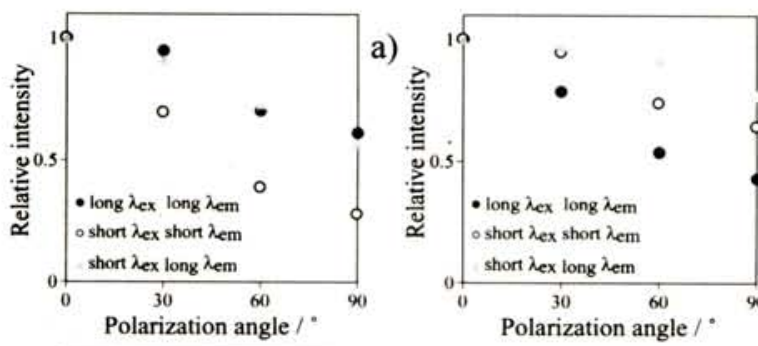

b)
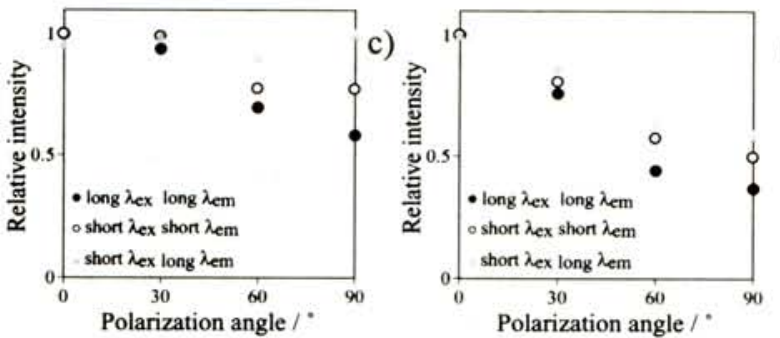

d)

Figure 12. Changes in UV-irrad-P4VPy(2b). Without (a)) and with (b)) correction of (at $\theta=\phi$ ), with corrections at $\theta(\mathrm{c})$ ), and with corrections at $\phi(\mathrm{d})$ ).

\section{Conclusions}

The polarization angle $(\theta, \phi)$ dependences of polarized fluorescence were determined and corrected for several cases of modified-PVPys(4) and UV-irrad-P4VPy (2) thin films.

For the correction procedures, instrumental 
functions were determined.

Emission intensities are calculated with changing polarization angles $(0 \leq \theta, \phi \leq 90)$ and some examples of mixed systems are also shown.

Emission bands of modified-PVPys(4) could be fitted to some of the mixed systems.

Changes in the emission intensities in the cases at $\theta=\phi, \theta$ or $\phi$, it has some possibilities to examine the nature of the first-excited state and fluoresce state in their photo-excitation processes.

i) One emission band appeared to increase with increasing $\theta(=\phi)$, which means the absence of $p$ component (in-plane) participation. As this was seen only for $\mathrm{P} 4 \mathrm{VPy}+\mathrm{FA}(\mathbf{4 c})+\mathrm{UV}$, large anion or accompanying networks could have an important role in the alignments of the transition moments.

ii) It is natural that most emission bands show in-plane alignment ( $p$-component), as most of the molecules were stretched with centrifugal force from center to the rim, when they were spin-coated. iii) Emission bands show various relationships with $\theta$ and / or $\phi$. With detailed analysis, the relationships among them would be clear.

From the differences of $\theta$ - and $\phi$-dependence of emission bands could be attributed to the distribution of $p$ - and $s$-components in the sample films. Therefore, the approximate distributions of transition moments could be estimated, even if no information of molecular complexes or aggregation is available.

We need more detailed study to establish the analysis. It would be necessary to examine other cases in which the fluorophore is known to have some in-plane and/or vertical alignment in films.

\section{Acknowledgement}

This work was supported by the 21 st. century's COE program organized by the Ministry of Education, Culture, Sports, Science and Technology (MEXT). S.N and Y.Y thanks to MEXT for their financial support.

\section{References}

1. I. F. Piérola, N. J. Turro, and P-L. Kuo, Macromol., 18, 508 (1985).

2. P. Cáceres and I.F. Piérola, Macromol. Chem. Rapid Commun., 8, 573 (1987).

3. I.F. Piérola, M. Cáceres, P. Cáceres, M.A. Castellanos, and J. Nuñez, Eur. Polym. J., 24, 895 (1988).

4. M. R. Gómez-Antón, J. G. Rodríguez, and I. F. Piérola, Macromol., 19, 2932 (1986).

5. P. Cáceres, P. Pascual, A. Horta, and I. F. Piérola,
Polym. Prepr., 28, 196 (1987).

6. T. Handa, Y. Utena, H. Yajima, and T. Ishii, $J$. Phys. Chem., 90, 2589 (1986).

7. T. Handa, Y. Utena, and H. Yajima, J. Phys. Chem., 88, 5150 (1984).

8. T. Handa, Y. Utena, H. Yajima, R. Katayama, T. Ishii, and T. Yamauchi, J. Phys. Chem., 90, 6324 (1986). 9. Y. Utena, H. Yajima, T. Ishii, and T. Handa, Eur. Polym. J., 23, 643 (1987).

10. Y. Utena, H. Yajima, T. Ishii, and T. Handa, Eur. Polym. J., 24, 71 (1987).

11. D. Vyprachtický, K.W. Sung, and Y. Okamoto, J. Polym. Sci. Pt.A: Polym. Chem., 37, 1341 (1999).

12. E. Vaganova, G. Meshulam, Z. Kotler, M. Rosenberg, and S. Yitzchaik, J. Fluorescence, 10, 81 (2000).

13. E. Vaganova, S. Yitzchaik, Polymeric Mater. Sci. Eng., 84, 1089 (2001).

14. E. Vaganova, S. Yitzchaik, 224th ACS Natl. Mtg (Boston, USA), Abst., POLY-058, (2004).

15. E. Vaganova, S. Yitzchaik, Macromol. Symp., 207, 95 (2004).

16. W.K. Fife, "Polymeric materials encyclo- pedia", J.C. Salamone et al. eds., CRC Press, Inc.: Boca Raton, pp. 7138 (1996), and references cited therein. 17. A.L. Weikel, S.D. Conklin, and J.N. Richardson, Sens. Actuators B: Chem., 110, 112 (2005).

18. B.J. Kim, J. Bang, C.J. Hawker, E.J. Kramer, Macrolmol., 39, 4108 (2006).

19. S. Nishiyama, M. Tajima, and Y. Yoshida, $J$. Photopolym. Sci. Tech., 16, 173 (2003).

20. S. Nishiyama, M. Tajima, and Y. Yoshida, $J$. Photopolym. Sci. Tech., 16, 671 (2003).

21. S. Nishiyama, M. Tajima, and Y. Yoshida, $J$. Photopolym. Sci. Tech., 17, 87 (2004).

22. S. Nishiyama, M. Tajima, and Y. Yoshida, $J$. Photopolym. Sci. Tech., 17, 727 (2004).

23. S. Nishiyama, M. Tajima, and Y. Yoshida, $J$. Photopolym. Sci. Tech., 18, 109 (2005).

24. S. Nishiyama, M. Tajima, and Y. Yoshida, $J$. Photopolym. Sci. Tech., 18, 683 (2005).

25. S. Nishiyama, M. Tajima, and Y. Yoshida, Thin Solid Films, 509, 177 (2006).

26. S. Nishiyama, M. Tajima, and Y. Yoshida, $J$. Photopolym. Sci. Tech., 19, 21 (2006).

27. S. Nishiyama, M. Tajima, and Y. Yoshida, $J$. Photopolym. Sci. Tech., 19, 727 (2006).

28. S. Nishiyama, M. Tajima, and Y. Yoshida, submitted to Colloid Surface A.

29. S. Nishiyama, M. Tajima, Y. Nakajima, T. Hanajiri, and Y. Yoshida, in preparation.

30. B. Valeur, "Molecular Fluorescence", WILEY-VCH Verlag GmbH, Weinheim (2002). 